\title{
Exercícios de fortalecimento para a reabilitação de lesão em ligamento cruzado
}

\section{anterior}

Strengthening exercises for rehabilitation of anterior cruciate ligament injury

Ejercicios de fortalecimiento para la rehabilitación de la lesión del ligamento cruzado anterior

Recebido: 26/10/2021 | Revisado: 05/11/2021 | Aceito: 08/11/2021 | Publicado: 13/11/2021

\author{
Sofia Silva Oliveira \\ ORCID: https://orcid.org/0000-0003-4598-2450 \\ Centro Universitário da Amazônia, Brasil \\ E-mail: sofiaoliveira2399@gmail.com \\ Leandro Alves Barros \\ ORCID: https://orcid.org/0000-0001-9695-034X \\ Centro Universitário da Amazônia, Brasil \\ E-mail: barrosleandroalves@gmail.com \\ Tainá Christielle Rocha Moraes \\ ORCID: https://orcid.org/0000-0001-6212-2132 \\ Centro Universitário da Amazônia, Brasil \\ E-mail: tainamoraes701@gmail.com \\ Wanderson Fernandes Silva \\ ORCID: https://orcid.org/0000-0001-9389-5795 \\ Centro Universitário da Amazônia, Brasil \\ E-mail:wandersonfsm@outlook.com
}

\begin{abstract}
Resumo
Objetivo: Analisar a eficácia de exercícios de fortalecimento para pacientes pós lesão de ligamento cruzado anterior através da análise de indicadores encontrados na literatura. Metodologia: Trata-se de uma revisão bibliográfica sistemática, tais dados utilizados na composição deste estudo foram coletados no banco de dados do site: PUBMED, e em revistas e livros periódicos que tenham a temática do estudo. Os documentos selecionados qual se referem ao tema, e que estão publicados nos anos 2016 a 2021 foram excluídos estudos de caso, estudos incompreensíveis, revisões sistemáticas e com a metodologia inadequada, e que foram descritos de forma incoesa e incoerente ao tema proposto Resultados: Nas buscas pelas literaturas, resultou em um achado de 1065 artigos usando as palavras chaves "anterior cruciate ligament, muscle strengtheing", porém apenas 185 se correlacionava ao tema de lesão de ligamento cruzado anterior, destes 185 apenas 6 correspondera a todos os critérios de inclusão e descrevia diretamente sobre alguma técnica de exercício de fortalecimento pós lesão de ligamento cruzado anterior, e na análise da escala Pedro, apresentou resultado acima de 7. Conclusão: Treinamentos de força progressiva é eficiente para recuperação do quadríceps e isquiotibiais, e os exercícios de resistência excêntrico de cadeia aberta, isocinético e concêntrico auxilia no fortalecimento e na ativação neuromuscular são eficientes para recuperação mesmo que em fase tardia, a intervenção através dos exercícios de fortalecimento associadas a outras terapêuticas para recuperação do quadro de pós LCA, aumenta os efeitos da reabilitação do paciente.
\end{abstract}

Palavras-chave: Ligamento; Cruzado anterior; Fortalecimento; Muscular.

\begin{abstract}
Objective: To analyze the effectiveness of strengthening exercises for patients after anterior cruciate ligament injury through the analysis of indicators found in the literature. Methodology: This is a systematic literature review, such data used in the composition of this study were collected in the website's database: PUBMED, and in journals and periodical books that have the theme of the study. The selected documents which refer to the theme, and which are published in the years 2016 to 2021, were excluded from case studies, incomprehensible studies, systematic reviews and with inadequate methodology, and which were described in an incohesive and inconsistent way to the proposed theme Results: Literature searches resulted in a finding of 1065 articles using the keywords "anterior cruciate ligament, muscle strengtheing", but only 185 correlated with the theme of anterior cruciate ligament injury, of these 185 only 6 met all the inclusion criteria and directly described some strengthening exercise technique after anterior cruciate ligament injury, and in the Pedro scale analysis, it presented a result above 7. Conclusion: Progressive strength training is efficient for quadriceps and hamstring recovery, and eccentric resistance exercises open-chain, isokinetic and concentric aids in strengthening and activating neu romuscular muscles are efficient for recovery even in a late phase, intervention through strengthening exercises associated with other therapies for recovery from the post ACL condition increases the effects of the patient's rehabilitation.
\end{abstract}

Keyword: Anterior; Cruciate; Ligament; Muscle; Strengtheing. 


\begin{abstract}
Resumen
Objetivo: Analizar la efectividad de los ejercicios de fortalecimiento en pacientes con lesión del ligamento cruzado anterior mediante el análisis de indicadores encontrados en la literatura. Metodología: Se trata de una revisión sistemática de la literatura, los datos utilizados en la composición de este estudio fueron recolectados en la base de datos del sitio web: PUBMED, y en revistas y libros periódicos que tienen la temática del estudio. Los documentos seleccionados que hacen referencia a la temática, y que se publican en los años 2016 a 2021, fueron excluidos de casos de estudio, estudios incomprensibles, revisiones sistemáticas y con metodología inadecuada, y que fueron descritos de manera incohesiva e inconsistente con la temática propuesta. Resultados: Las búsquedas bibliográficas dieron como resultado un hallazgo de 1065 artículos que usaban las palabras clave "ligamento cruzado anterior, fortalecimiento muscular", pero solo 185 se correlacionaron con el tema de la lesión del ligamento cruzado anterior, de estos 185 solo 6 cumplieron con todos los criterios de inclusión y describieron directamente algún fortalecimiento. técnica de ejercicio después de una lesión del ligamento cruzado anterior, y en el análisis de la escala de Pedro, presentó un resultado por encima de 7. Conclusión: El entrenamiento de fuerza progresivo es eficiente para la recuperación de cuádriceps e isquiotibiales, y ejercicios de resistencia excéntrica de cadena abierta, ayudas isocinéticas y concéntricas en el fortalecimiento y activando neu Los músculos musculares son eficientes para la recuperación incluso en una fase tardía, la intervención a través de ejercicios de fortalecimiento asociados con otras terapias para la recuperación de la condición posterior al LCA aumenta los efectos de la rehabilitación del paciente.
\end{abstract}

Palabras clave: Ligamento; Cruzado; Anterior; Fortalecimento; Muscular.

\title{
1. Introdução
}

A lesão de ligamento pode ser considerada comum em indivíduos fisicamente ativos, sendo o ligamento cruzado anterior (LCA) um dos principais ligamentos do joelho, responsável por 86\% da estabilização anterior da articulação (Castro e Acras, 2011). Situado no complexo funcional do joelho classificado como articulação do tipo gínglimo, originando-se no encontro das articulações patelofemoral e tibiofemoral, onde permite realizar movimentos angulares de flexão, extensão e quando está em semi-flexão realizar rotações, por esta gama de movimentos é uma das articulações mais lesionada por exposição de forças externas, fato comum no meio esportivo, com o público jovem, como maiores afetados (Soares, et al., 2011). Devido a articulação mesmo após a lesão não cicatrizar adequadamente por diversos fatores, há um índice muito alto de cirurgias para a reconstrução do referido ligamento (Arliani, et al., 2012). O tratamento cirúrgico em casos de lesão do ligamento cruzado anterior é um dos meios de tratamento invasivo e é indicado quando todas as fibras se rompem, e causa na articulação dor, instabilidades na marcha, e anteriorização da tíbia (Fukuda, 2020).

Fatores epidemiológicos mostram que cerca de $80 \%$ das lesões do LCA acontecem sem trauma direto, instigando assim a ciência buscar fatores de risco de caráter, tanto intrínsecos como extrínsecos (Silva, et al., 2012), pode-se citar como fatores de riscos para esta lesão, excesso de translação anterior da tíbia em relação ao fêmur, as mudanças cinemáticas no tronco, na pelve e no quadril e alterações da força de recrutamento de músculos estabilizadores do complexo lombo pélvico pode favorecer a ocorrência de um valgismo, produzindo assim um estresse muito grande no ligamento, causando um som audível no momento da entorse, seguida de dor incapacitante, deixando propenso a lesão, principalmente em mulheres (Araújo e Pinheiro, 2015). Quando existe a ruptura de um dos ligamentos do joelho, pode-se tornar instável e gerar episódios de falseio e edema, a ruptura deste ligamento ocasiona respostas neuromusculares e biomecânicas complexas. (Prado, 2013), as lesões de ligamentos estão classificadas em três graus:

Grau 1 - Lesão do tipo leve, onde há edema e sensibilidade local, com rompimento de algumas fibras sem perda funcional.

Grau 2 - Lesão do tipo moderada, onde grande parte das fibras encontram-se rompidas, seguido de instabilidade na articulação, não demostrando perda completa da integralidade do ligamento.

Grau 3 - Lesão do tipo grave, acontecendo o rompimento completo do ligamento.

A ruptura do ligamento cruzado anterior geralmente ocorre após um trauma torcional do joelho, o mais comum ocorre quando paciente roda o seu corpo e o seu pé permanece fixo no chão, sendo comuns em atividades esportivas em que o joelho faz movimento de rotação, como no futebol, basquete e esqui. (Astur, et al., 2013) apresentaram incidência de lesão 53,27\% no 
futebol, 6,54\% nas atividades de corrida, 5,6\% nas atividades de academia, 4,67\% no voleibol, ciclismo e surfe. Outras modalidades menos comuns foram jiu-jitsu, capoeira, natação, caminhada, triatlon e dança; 80,37\% das lesões isoladas do LCA no esporte foram em homens no futebol essa incidência subiu para 87,72\% (Astur, et al., 2016). Devido á grande incidência de dano ao LCA, diversos estudos foram publicados sob esta temática, e vem a ser o ligamento mais estudado do corpo humano (Frobell, et al., 2010).

A reabilitação na lesão do ligamento cruzado anterior, é realizada individualmente, pois deve se adequar ao quadro de baixo ou alto risco de cada paciente, em caso de baixo risco o tratamento é feito de maneira conservadora, já nos casos de alto risco, onde há um risco de instabilidade, e consequentemente a incidência de uma nova lesão, o tratamento deve ser cirúrgico (Pinheiro, 2015). A fisioterapia é fundamental na reabilitação do pós-cirúrgico de LCA, pois agiliza o retorno das atividades diárias no menor tempo possível e com maior segurança, e atua neste caso, no fortalecimento, alívio da dor, no ganho de amplitude de movimento, equilíbrio, propriocepção e demais funções. O presente estudo de revisão bibliográfica, objetiva analisar e reunir os principais protocolos utilizados no fortalecimento do ligamento cruzado anterior através de exercícios no processo de reabilitação após a realização de cirurgia.

\section{Metodologia}

A metodologia se trata de uma descrição formal dos métodos e técnicas que foram usados para seleção dos artigos para a pesquisa, também revelam as conexões que o autor utilizou para o quadro teórico e seus objetos na pesquisa. (Minayo,2010). Os métodos não são únicos, existem uma gama de métodos que visam suprir as necessidades de acordo com o assunto e a finalidade proposto de cada pesquisa. (Aragão e Neta,2017).

Este estudo trata-se de revisão sistemática, foi descrito por três integrantes de um grupo de trabalho de conclusão de curso, a coleção dos artigos de base fora realizada de forma independente na fonte da base de dados do pubmed, e as discordâncias ou qualquer divergência para seleção dos artigos foram solucionadas através de ligação ou vídeo chamada. As buscas pelos estudos ocorrerão no período de abril até setembro de 2021, utilizando as palavras chaves: anterior; cruciate; ligament; muscle; strengtheing;.Os documentos selecionados foram os que se referiam ao tema, publicados no período dos anos de 2016 a 2021, nos idiomas oficial do país (Português) e estrangeiros (Inglês), ensaios clínicos randomizados, de pontuação na escala Pedro maior que 7, que estivessem relacionados aos exercícios de fortalecimento após cirurgia de LCA, foram excluídos artigos de estudos de caso, estudos incompreensíveis, revisões sistemáticas, e projetos com metodologia inadequada.

\section{Resultados e Discussão}

O presente estudo teve como objetivo principal analisar a eficácia de exercícios de fortalecimento muscular no manejo de ligamento cruzado anterior, para tanto a busca realizada nas bases de dados do Pubmed com as palavras chaves "anterior cruciate ligament, muscle strengtheing" resultou em um total de achados de 1065 artigos, a inclusão dos artigos selecionados foram aqueles que cumpriram os critérios: Devidamente ligados aos tema de fortalecimento muscular $(=6)$,serem ensaios clínicos (=94), ou testes controlados e aleatórios $(=82)$ e livros ou documentos(=3). Foram excluídos artigos de revisão sistemática (=85), meta análises $(=40)$ análises $(=77)$, estudo de caso $(=5)$, e demais artigos que não cumpria nenhuma das propostas dos critérios de inclusão descritos acima e não estavam relacionados ao tema proposto (=637). Dentre os artigos analisados e aceitos pelos critérios de inclusão estabelecidos, um total de 6 estudos foram selecionados para composição deste trabalho. A amostra dos 6 artigos encontrados variou de 20 a 68 participantes com pelo menos 2 dois grupos em cada estudo, com exceção do estudo de (Zebis, et al., 2019) no qual apresentou apenas um grupo. Quanto a intervenção observa-se que todos os estudos em pelo menos um grupo utilizou-se de alguma forma de fortalecimento muscular para o manejo das lesões 
de LCA, outras terapêuticas utilizadas foram: alongamentos e crioterapia (Kuenze, et al., 2016), exercício com peso corporal (Zebis, et al., 2019), treinamento de exercícios excêntricos e isocinéticos (Vidmar, et al., 2019), exercícios combinados de resistência (Bregenhof, et al., 2019). Quanto a duração do tratamento variou-se entre 14 sessões uma vez por dia (Kuenze, et al., 2016), 6 e 12 semanas, e o protocolo de tratamento maior de 10 meses de treinamento de força no estudo (Welling, et al., 2019,). Foram observadas como análises, a eletromiografia no estudo de (Zebis, et al., 2019), e teste de contração isométrica voluntária máxima para os outros estudos (Harput, et al., 2018, Kuenze, et al., 2016). Outras variáveis analisadas foram os índices de simetria de membro no estudo de (Welling, et al., 2019,).), ressonância magnética, dinamometria e o questionário do formulário subjetivo de joelho (IKDC) de (Vidmar, et al., 2019), Tegner Activity Score, e a taxa de desenvolvimento de força (RFD) realizada no estudo de (Bregenhof, et al., 2019), e teste de explosão de força para musculatura do quadríceps (Kuenze, et al., 2016).

Os estudos tiveram pontuação que variou entre 7 a 11 pontos da Escala Pedro, todos os artigos cumprirão os itens 1, 7, 8 designados pela escala, em relação ao item 2, foram observados que apenas em (Welling, et al., 2019), não se enquadrou, pois não se tratava de um estudo que os participantes foram distribuídos aleatoriamente, mesmo porque, todos participaram estavam em um grupo unitário, o qual receberam o mesmo protocolo de tratamento. Em relação ao item 3 da Escala Pedro, apenas (Zebis, et al., 2019) não cumpriu o requisito de alocação secreta dos sujeitos, pois todos estavam cientes do qual protocolo de tratamento ao qual se submeteria, antes de começar a pesquisa e que fariam parte de um único grupo. O requisito do item 5 da escala Pedro que se referia aos sujeitos participarem de forma cega de toda pesquisa, não foi observada em (Zebis, et al., 2019, Vidmar, et al., 2019, Kuenze, et al., 2016), deste estudo. O item 6, não foi observada em (Zebis, et al., 2019, Harput, et al., 2018, Vidmar, et al., 2019, Kuenze, et al., 2016), pois em ambas pesquisas, os terapeutas trabalhavam de forma consciente e eram sabedores a qual grupo estes participantes estavam designados.

De acordo com o item 10, que se refere a comparação estatística entre os grupos, ou entre os tratamentos, pode-se observar que isso não foi descrito em (Harput, et al., 2018, Bregenhof, et al., 2019), e o item 11, não foi descrito em (Kuenze, et al., 2016), os resultados não foram descritos por uma medida de dimensão do efeito do tratamento.

A análise descrita pode ser observada na Tabela 1:

Tabela 1. Características dos estudos selecionados.

\begin{tabular}{|c|c|c|c|c|c|c|c|c|}
\hline Autor & Amostra & Grupos de estudo & Intervenção & $\begin{array}{l}\text { Duração de } \\
\text { tratamento }\end{array}$ & $\begin{array}{l}\text { Variáveis } \\
\text { estudadas }\end{array}$ & $\begin{array}{c}\text { Pontuação } \\
\text { Pedro }\end{array}$ & Resultados & Conclusões \\
\hline $\begin{array}{l}\text { ZEBIS, et } \\
\text { al. } 2019\end{array}$ & 20 & Grupo unitário & $\begin{array}{l}\text { Exercício de } \\
\text { peso corporal }\end{array}$ & $\begin{array}{l}\text { 2x por semana } \\
\text { em } 4 \text { semanas }\end{array}$ & $\begin{array}{c}\text { eletromiografia / } \\
\text { teste de } \\
\text { contração } \\
\text { isométrica } \\
\text { voluntária } \\
\text { máxima }\end{array}$ & 7 & $\begin{array}{c}\text { Não houve } \\
\text { diferenças } \\
\text { significativas no } \\
\text { ganho de força } \\
\text { muscular. }\end{array}$ & $\begin{array}{l}\text { Aumento da } \\
\text { atividade do } \\
\text { quadríceps. }\end{array}$ \\
\hline $\begin{array}{l}\text { WELLIN } \\
\text { G, et al. } \\
2019\end{array}$ & 68 & $\begin{array}{l}\text { Experimental / } \\
\text { Grupo Controle }\end{array}$ & $\begin{array}{c}\text { Força isocinética } \\
\text { / Treinamento de } \\
\text { força }\end{array}$ & 10 meses & $\begin{array}{c}\text { Índice de simetria } \\
\text { de membro (LSI)> } \\
90 \%\end{array}$ & 11 & $\begin{array}{l}\text { Sem diferenças } \\
\text { significativas } \\
\text { no pico de } \\
\text { força entre os } \\
\text { grupos. }\end{array}$ & $\begin{array}{l}\text { O princípio da } \\
\text { força } \\
\text { progressiva, } \\
\text { foi efetivo na } \\
\text { recuperação de } \\
\text { quadríceps e } \\
\text { isquiotibiais }\end{array}$ \\
\hline $\begin{array}{l}\text { HARPUT, } \\
\text { et al. } 2018\end{array}$ & 48 & $\begin{array}{l}\text { G1: concêntrico } \\
\text { Com reabilitação G2: } \\
\text { excêntrico com } \\
\text { reabilitação. } \\
\text { G3: Controle }\end{array}$ & $\begin{array}{l}\text { Educação } \\
\text { cruzada (CE) } \\
\text { concêntrica e } \\
\text { excêntrica no } \\
\text { quadríceps }\end{array}$ & 12 semanas & $\begin{array}{l}\text { Teste de } \\
\text { contração } \\
\text { isométrica } \\
\text { voluntária } \\
\text { máxima }\end{array}$ & 9 & $\begin{array}{c}\text { A força do } \\
\text { quadríceps de } \\
\text { ambos os joelhos } \\
\text { foi maior nos } \\
\text { grupos } \\
\text { concêntrico e } \\
\text { excêntrico. }\end{array}$ & $\begin{array}{l}\text { Programa de } \\
\text { exercícios } \\
\text { concêntricos e } \\
\text { isocinéticos } \\
\text { observa } \\
\text { melhora nas } \\
\text { CIVMS. }\end{array}$ \\
\hline
\end{tabular}




\begin{tabular}{|c|c|c|c|c|c|c|c|c|}
\hline $\begin{array}{l}\text { BREGEN } \\
\text { HOF, et al. } \\
2019\end{array}$ & 50 & $\begin{array}{l}\text { Grupo experimentale } \\
\text { grupo controle }\end{array}$ & $\begin{array}{l}\text { Exercícios } \\
\text { combinados } \\
\text { de resistência } \\
\text { progressiva e } \\
\text { neuromuscul } \\
\text { ar na força } \\
\text { flexora / } \\
\text { extensora do } \\
\text { joelho }\end{array}$ & 12 semanas & $\begin{array}{l}\text { Tegner Activity } \\
\text { Score, a taxa de } \\
\text { desenvolvimento } \\
\text { de força (RFD) }\end{array}$ & 9 & $\begin{array}{l}\text { Redução da força } \\
\text { dos isquiotibiais } \\
\text { representa risco } \\
\text { potencial para } \\
\text { ruptura secundária } \\
\text { do LCA. }\end{array}$ & $\begin{array}{l}\text { Combinaçã } \\
\text { o eficaz do } \\
\text { treinamento } \\
\text { de } \\
\text { resistência } \\
\text { progressiva, } \\
\text { e exercícios } \\
\text { neuromuscu } \\
\text { lares na } \\
\text { fase de } \\
\text { reabilitação } \\
\text { tardia. }\end{array}$ \\
\hline $\begin{array}{l}\text { VIDMAR, } \\
\text { et al. } 2019\end{array}$ & 30 & $\begin{array}{c}\text { Grupo convencional e } \\
\text { Grupo isocinético }\end{array}$ & $\begin{array}{l}\text { Treinamento } \\
\text { excêntrico e } \\
\text { isocinético }\end{array}$ & 6 semanas & $\begin{array}{c}\text { Ressonância } \\
\text { magnética, } \\
\text { dinamometria, } \\
\text { questionário }\end{array}$ & 9 & $\begin{array}{l}\text { Treinamento } \\
\text { excêntrico e } \\
\text { isocinético, } \\
\text { geram melhores } \\
\text { resultados. }\end{array}$ & $\begin{array}{c}\text { O uso do } \\
\text { exercício } \\
\text { excêntrico de } \\
\text { cadeia aberta } \\
\text { sendo eficaz } \\
\text { para } \\
\text { reabilitação } \\
\text { muscular de } \\
\text { quadríceps. }\end{array}$ \\
\hline $\begin{array}{l}\text { KUENZE, } \\
\text { et al. } 2016\end{array}$ & 20 & $\begin{array}{c}\text { Grupos pós } \\
\text { reconstrução de LCA } \\
\text { primário unilateral / } \\
\text { participante } \\
\text { saudáveis. }\end{array}$ & $\begin{array}{c}5 \text { min } \\
\text { caminhada } \\
>4 \mathrm{~km} / \mathrm{h}, \\
\text { agachamento } \\
\text { s com salto, } \\
\text { saltos } \\
\text { laterais, } \\
\text { extensão } \\
\text { joelho, teste } \\
\text { de explosão, } \\
\text { crioterapia, } \\
\text { alongamento, } \\
\text { exercícios de } \\
\text { fortaleciment } \\
\text { o } \\
\text { progressivo, } \\
\text { treinamento } \\
\text { de equilíbrio. }\end{array}$ & $\begin{array}{c}1 \text { hora por dia, } \\
\text { sendo feita } 14 \\
\text { sessões }\end{array}$ & $\begin{array}{c}\text { Teste contração } \\
\text { isométrica } \\
\text { voluntária } \\
\text { máxima } \\
\text { normalizada de } \\
\text { extensão de } \\
\text { joelho (CIVM) e } \\
\text { teste de explosão } \\
\text { sobreposta de } \\
\text { quadríceps antes } \\
\text { e } \\
\text { após protocolo } \\
\text { de exercícios } \\
\text { fatigantes de } 30 \\
\text { minutos }\end{array}$ & 8 & $\begin{array}{c}2 \text { semanas de } \\
\text { fortalecimento do } \\
\text { quadríceps } \\
\text { reduziram a } \\
\text { diferença entre os } \\
\text { grupos. }\end{array}$ & $\begin{array}{c}\text { Fortalecimen } \\
\text { to do } \\
\text { quadríceps } \\
\text { indicou } \\
\text { restauração } \\
\text { das funções } \\
\text { neuro } \\
\text { muscular } \\
\text { com aumento } \\
\text { da ativação } \\
\text { durante as } \\
\text { atividades } \\
\text { físicas. }\end{array}$ \\
\hline
\end{tabular}

\footnotetext{
* LSI (índice de simetria de membros), CE (educação cruzada), CIVMS (força isométrica voluntaria máxima do quadríceps), RFD (taxa de desenvolvimento de força), LCA (ligamento cruzado anterior).

Fonte: Oliveira, Barros, Moraes e Silva (2021).
}

Inúmeras técnicas fisioterapêuticas são encontradas na literatura sobre exercícios de fortalecimento pós-operatório de LCA, podemos verificar que dentro dos últimos 5 anos foram observadas algumas técnicas obtidas como "melhores práticas", analisados quanto sua eficácia. O estudo de (Zebis, et al., 2019) apresentou o objetivo de recuperar a força muscular após lesão de LCA através do controle de intensidade dos exercícios na reabilitação, avaliou a progressão da atividade dos músculos isquiotibiais e quadríceps especialmente, o músculo quadríceps medial (vasto medial) durante os exercícios que faziam uso apenas do peso corporal, contabilizou um total de 20 participantes atletas saudáveis de idades não informadas, tendo apenas um grupo unitário. O protocolo de reabilitação foi dividido em blocos (fase 1 e 2 / fase 4 e 5 / fase 3 ) com cinco objetivos (amplitude de movimento, função muscular, sintomas, função de caminhar e equilíbrio / coordenação) resultando em um tempo total de 24 semanas, durante a seleção dos exercícios em cada fase foi selecionado 03 exercícios, sendo um com predominância de quadríceps, outro com isquiotibiais e outro misto totalizando assim 9 exercícios usados, todos os exercícios foram avaliados através da eletromiografia. O referente estudo evidenciou a necessidade de um consenso na seleção de exercícios e dose para isquiotibiais focando sua progressão, apresentando um contraste com o quadríceps que teve aumento 
progressivo de uma fase para a outra. (Kilgas, et al., 2019), em seu estudo realiza a comparação dos resultados de um protocolo de treinamento de força, para reabilitação de quadríceps e isquiotibiais, com dois grupos de atletas: experimental e controles saudáveis, busca também averiguar seus efeitos no pico de força nestes músculos e função do joelho. Este protocolo, foi aplicado no período de 10 meses para os participantes do grupo experimental e os participantes do grupo controle, realizaram os testes uma única vez em um dia de treinamento. O protocolo de fortalecimento dos isquiotibiais foi aplicado em 4 fases, o avanço para a próxima fase se designava mediante cumprimento dos critérios estabelecidos pelos testes. A primeira fase se resumiu em exercícios de ganho de ADM total de joelho, redução do quadro álgico, e ativação da musculatura do quadríceps. A segunda fase consistia em cinesioterapia com os exercícios no step, elevação das pernas e leg press, porém ainda, sem aplicação de exercícios de cadeia cinética aberta. Mediante a estabilidade dos participantes ao teste isocinético de força, foi realizado a terceira fase do fortalecimento, nessa fase a progressão dos exercícios incide em melhora da força e regularização da simetria da força do membro, foram incluídos a esta fase, exercícios de cadeia cinética aberta e fechada com resistência, exercícios de equilíbrio, realizando as atividades para ambos os membros e avançando para adm completa de joelho, ao final realizando a segunda sessão de testes, para prosseguimento da fase quatro. Nesta última fase de treinamento, foi adaptado os exercícios baseando-se nos resultados dos testes isocinéticos, como forma de reajuste para melhorar a simetria de força utilizando os mesmos exercícios das fases anteriores, com variações caso fosse necessário. Encerrando a quarta fase, e realizado a sessão de testes isocinéticos, qualquer alteração de déficit de força obtida, foi reajustado com o treinamento de força e resistência. Em uma análise, aos 4 meses de tratamento foi constatado pico de força muscular do quadríceps menor no grupo experimental comparado ao grupo controle. Aos 7 meses de tratamento o pico de força em ambos os grupos não havia grandes diferenças, em quadríceps e isquiotibiais, e em 10 meses de treinamento, o grupo experimental apresentou maior pico de força de isquiotibiais do que o grupo controle. (Harput, et al., 2018), apresenta um estudo sobre educação cruzada como melhora da recuperação da força do quadríceps, e funcionalidade do joelho após ter sido realizado a reconstrução do ligamento cruzado anterior (LCA), os participantes foram divididos aleatoriamente em três grupos de treinamento, o primeiro grupo usava como método exercícios concêntricos com reabilitação, o segundo grupo utilizava exercícios excêntricos como reabilitação, o terceiro grupo realizava apenas exercícios de reabilitação, a força do quadríceps foi determinada a partir da definição da contração isométrica voluntaria máxima (CIVM) do quadríceps isométrico para o mesmo foi utilizado um dinamômetro isocinético, neste teste os participantes realizaram três CIVM com duração de 5 segundos e descanso de 2 minutos entre cada CIVM, a função do joelho foi determinada através de um teste de salto de uma perna e de um formulário subjetivo do joelho, o programa de reabilitação foi realizado por todos os participantes, e teve duração de 24 semanas, onde os participantes realizavam a fisioterapia 3 vezes por semana até a 12 semana, e treinamento neuromuscular progressivo, treino de equilíbrio e exercícios de fortalecimento, na 12 semana os participantes foram orientados a realizar os exercícios em casa até a 24 semana, os grupos 1 e 2 participaram de exercícios isocinéticos concêntricos ou excêntricos do membro contralateral 3 vezes por semana durante 8 semanas, em cada sessão de exercícios, eram feitas séries de 12 repetições e período de 2 minutos de descanso entre cada exercício. Em 4 semanas a força do quadríceps era igual entre os 3 grupos, na 12 semana a força do quadríceps foi maior nos grupos 1 e 2, e entre o grupo 1 e 2 não houve diferença significativa da força, na 24 semana apenas o 1 grupo teve uma força maior comparado com os outros dois grupos. (Bregenhof, et al., 2019) em seu estudo investiga os efeitos de exercícios combinados, progressivos de força, e neuromuscular do joelho, e a capacidade funcional dos isquiotibiais em pacientes reconstruídos com LCA, que apresentam deficiência de força muscular nestes músculos, e em fase de reabilitação tardia (12-24 meses),para este estudo participaram uma mostra de 50 pacientes com idades entre 18 e 40 anos divididos em dois grupos sendo um grupo controle que realizaram exercícios domiciliares duas vezes na semana usando do peso corporal e das faixas de resistência, e outro grupo experimental, realizando exercícios de força combinada e intervenção de exercícios neuromusculares envolvidos em um regime de exercícios baseado em treinamento de força progressiva, incluindo elementos 
de exercícios neuromusculares, todo o protocolo foi baseado em exercícios descritos na literatura, tiveram prática duas vezes na semana durante 12 semanas tendo sessões de 60-70 minutos. Ao final todos os pacientes foram avaliados pela dinamômetria, E pode-se perceber que essas estratégias de intervenção são eficazes aos pacientes de reconstituição de LCA tardio. No estudo de (Vidmar, et al., 2019), os participantes foram separados em dois grupos, cada um recebeu um protocolo de tratamento específico, antes de serem realocados, os participantes foram submetidos ao processo de reabilitação padrão de fisioterapia, como nos estudos anteriores, com o objetivo inicial de alívio de dor, ganho de ADM, evitar trofismo muscular, ganho de equilíbrio, atividade neuromuscular, propriocepção entre outros. Os participantes do grupo isocinético receberam protocolos de treinamento isocinético excêntrico de extensão máxima de joelho, resistindo ao movimento flexor do dinamômetro, estes exercícios foram aplicados por 6 semanas, duas vezes por semana com intervalo de descanso de 1 minuto. Já o treinamento do grupo convencional, utilizou-se de uma cadeira extensora. Para este exercício o terapeuta auxiliava na fase concêntrica de $90^{\circ}$ a $30^{\circ}$, e orientava ao paciente o retorno do movimento com as pernas para $90^{\circ}$. A carga era aumentada de acordo com o feedback e força muscular do paciente. A duração do treinamento do grupo convencional foi o mesmo do grupo isocinético. Nos resultados gerais de força o grupo isocinético apresentou melhorias tanto em pré como em pós treinamentos em todos os critérios da pesquisa do que o grupo convencional (Kuenze, et al., 2016), em seu estudo busca avaliar o impacto de um programa de fortalecimento do quadríceps no período de 2 semanas em pacientes com histórico de LCA, para isso foi selecionado dois grupos cada um com 10 participantes, foram divididos em pacientes com histórico de LCA e controle saudáveis. Ao início do tratamento os pacientes precisariam realizar os testes de contração isométrica voluntária máxima normalizada da extensão do joelho (CIVM) e o teste de explosão sobreposta do quadríceps ao qual ambas as equipes conseguiram realizar, logo após foi aplicado um protocolo de exercícios fatigantes durante 30 minutos, finalizando novamente com os testes que foram realizados no início do tratamento. Os exercícios fatigantes compreendiam-se em caminhada na esteira de 5 minutos, séries de agachamentos, saltos laterais. No treinamento com o dinamômetro foram realizados 2 testes de prática de extensão de joelho isométrica submáxima e 2 testes de extensão isométrica máxima de joelho. Além dos treinamentos de fortalecimento foi realizado com os pacientes crioterapia no joelho, alongamento de MMII, treinamento de equilíbrio, e treinamento de progressão. Obtendo como resultado melhora significativa do torque normalizado de extensão de joelho e também de ativação central do quadríceps.

\section{Conclusão}

Os resultados encontrados mostram que fazendo uso dos princípios dos treinamentos de força progressiva é eficiente para recuperação do quadríceps e isquiotibiais, musculaturas tais, que são essências para o controle do movimento do joelho, bem como também exercícios de resistência excêntrico de cadeia aberta, isocinético e concêntrico auxilia no fortalecimento e na ativação neuromuscular são eficientes para recuperação mesmo que em fase tardia, a intervenção através dos exercícios de fortalecimento associadas a outras terapêuticas para recuperação do quadro de pós LCA, aumenta os efeitos da reabilitação do paciente. Sugere-se que novas pesquisas sejam realizadas com intuito de aprofundamento do tema, contribuindo para as práticas clínicas e o manejo com o processo de reabilitação de pacientes com lesão de ligamento cruzado anterior.

\section{Referências}

Araujo, A. G. D. S., \& Pinheiro, I. (2015). Protocolos de tratamento fisioterápico nas lesões de ligamento cruzado anterior após ligamentoplastia-uma revisão. Cinergis. 16(1):61-65. https://online.unisc.br/seer/index.php/cinergis/article/view/5619. DOI: https://doi.org/10.17058/cinergis.v16i1.5619

Aragão, J. W. M. D., \& Mendes Neta, M. A. H. (2017). Metodologia científica. [e-book]. Edufba. http://repositorio.ufba.br/ri/handle/ri/30900

Arliani, G. G., Astur, D. C., Moraes, E. R., Kaleka, C. C., Jalikjian, W., Golano, P., \& Cohen, M. (2012). Three dimensional anatomy of the anterior cruciate ligament: a new approach in anatomical orthopedic studies and a literature review. Open access journal of sports medicine, 3, $183-188$. https://doi.org/10.2147/OAJSM.S37203 
Research, Society and Development, v. 10, n. 14, e533101422354, 2021

(CC BY 4.0) | ISSN 2525-3409 | DOI: http://dx.doi.org/10.33448/rsd-v10i14.22354

Astur, D. C., Batista, R. F., Arliani, G. G., \& Cohen, M. (2013). Tendências no tratamento das lesões do ligamento cruzado anterior do joelho nos sistemas de saúde público e privado do Brasil. São Paulo Medical Journal, 131 , 257-263.

Astur, D. C., Xerez, M., Rozas, J., Debieux, P. V., Franciozi, C. E., \& Cohen, M. (2016). Lesões do ligamento cruzado anterior e do menisco no esporte: impacto, tempo de prática até a lesão e limitações causadas pelo trauma 光. Revista brasileira de ortopedia, 51, 652-656.

Bregenhof, B., Jørgensen, U., Aagaard, P., Nissen, N., Creaby, M. W., Thorlund, J. B., Jensen, C., Torfing, T., \& Holsgaard-Larsen, A. (2018). O efeito do exercício direcionado na função muscular do joelho em pacientes com deficiência persistente dos isquiotibiais após a reconstrução do LCA - protocolo de estudo para um ensaio clínico randomizado. Trials , 19 (1), 75. https://doi.org/10.1186/s13063-018-2448-3

Castro, R. L. B. D. e Acras, S. D. (2011). Avaliação dos resultados da reconstrução do ligamento cruzado anterior com tendões flexores e transverso de guia rígido. Revista Brasileira de Ortopedia, 46, 143-147.

Santos, S., M., Marques, R. L., Frazão, R. S., \& Oliveira, A. T. (2011). Intervenção fisioterapêutica no pós-operatório de lesões do ligamento cruzado anterior. TEMA-Revista Eletrônica de Ciências (ISSN 2175-9553), 11(16).

Frobell, R. B., Roos, E. M., Roos, H. P., Ranstam, J., \& Lohmander, L. S. (2010). Um ensaio clínico randomizado de tratamento para rupturas agudas do ligamento cruzado anterior. New England Journal of Medicine , 363 (4), 331-342.

Fukuda, T. (2020). Lesão do ligamento cruzado anterior. Instituto trata. https://www.institutotrata.com.br/ligamento-cruzado-anterior/

Harput, G., Ulusoy, B., Yildiz, T. I., Demirci, S., Eraslan, L., Turhan, E., \& Tunay, V. B. (2019). Cross-education improves quadriceps strength recovery after ACL reconstruction: a randomized controlled trial. Knee surgery, sports traumatology, arthroscopy: official journal of the ESSKA, 27(1), 68-75. https://doi.org/10.1007/s00167-018-5040-1

Kuenze, C., Eltoukhy, M., Kelly, A., \& Kim, C. Y. (2017). Impacto do fortalecimento do quadríceps na resposta a exercícios fatigantes após a reconstrução do LCA. Journal of science and medicine in sport , 20 (1), 6-11.

Minayo, M. C. S. (Org.) Pesquisa social: teoria, método e criatividade. 29. ed. Rev. Atual. Petrópolis: Vozes, 2010.

Pinheiro, A., \& Sousa, C. V. (2015). Lesão do ligamento cruzado anterior: apresentação clínica, diagnóstico e tratamento. Rev Port Ortop Traum, 23(4), 320329

Prado, M. P., Fernandes, T. D., Camanho, G. L., Mendes, A. A. M., \& Amodio, D. T. (2013). Instabilidade mecânica pós-lesão ligamentar aguda do tornozelo. Comparação prospectiva e randomizada de duas formas de tratamento conservador. Revista Brasileira de Ortopedia , 48, $307-316$.

Silva, R. S. D., Ferreira, A. L. G., Veronese, L. M., Driusso, P., \& Serrão, F. V. (2012). Relação entre hiperpronação subtalar e lesões do ligamento cruzado anterior do joelho: revisão de literatura. Fisioterapia em Movimento, 25, 679-688

Vidmar, M. F., Baroni, B. M., Michelin, A. F., Mezzomo, M., Lugokenski, R., Pimentel, G. L., \& Silva, M. F. (2020). Isokinetic eccentric training is more effective than constant load eccentric training for quadriceps rehabilitation following anterior cruciate ligament reconstruction: a randomized controlled trial. Brazilian journal of physical therapy, 24(5), 424-432. https://doi.org/10.1016/j.bjpt.2019.07.003

Welling, W., Benjaminse, A., Lemmink, K., Dingenen, B., \& Gokeler, A. (2019). Progressive strength training restores quadriceps and hamstring muscle strength within 7 months after ACL reconstruction in amateur male soccer players. Physical therapy in sport : official journal of the Association of Chartered Physiotherapists in Sports Medicine, 40,10-18. https://doi.org/10.1016/j.ptsp.2019.08.004

Yu, M., Ao, Y. F., Wang, J. Q., Yong, M. A., Zhang, X., Wang, J. N., \& Zhu, J. X. (2013). Características clínicas de 4355 pacientes com lesão do ligamento cruzado anterior. Jornal médico chinês, 126 (23), 4487-4492.

Zebis, M. K., Sørensen, M. H., Lauridsen, H. B., Bencke, J., Andersen, C. H., Carlsbæk, J. B., Jespersen, P., Kallehauge, A. H., \& Andersen, L. L. (2019). Electromyography Evaluation of Bodyweight Exercise Progression in a Validated Anterior Cruciate Ligament Injury Rehabilitation Program: A CrossSectional Study. American journal of physical medicine \& rehabilitation, 98(11), 998-1004. https://doi.org/10.1097/PHM.0000000000001232 